\title{
The Effect of a Universal Cervical Length Screening Program on Antepartum Management and Birth Outcomes
}

\author{
Scott A. Shainker, DO, MS ${ }^{1,2}$ Anna M. Modest, $\mathrm{MPH}^{1,3}$ \\ Steven J. Ralston, MD, MPH ${ }^{1,2}$ \\ ${ }^{1}$ Department of Obstetrics, Gynecology and Reproductive Biology, \\ Harvard Medical School, Boston, Massachusetts \\ ${ }^{2}$ Division of Maternal-Fetal Medicine, Department of Obstetrics and \\ Gynecology, Beth Israel Deaconess Medical Center, Boston, \\ Massachusetts \\ ${ }^{3}$ Department of Obstetrics and Gynecology, Beth Israel Deaconess \\ Medical Center, Boston, Massachusetts
}

\begin{abstract}
Address for correspondence Steven J. Ralston, MD, MPH, Division of Maternal-Fetal Medicine, Department of Obstetrics and Gynecology, Beth Israel Deaconess Medical Center, 330 Brookline Avenue, Boston, MA 02215 (e-mail: sralston@bidmc.harvard.edu).
\end{abstract}

Am J Perinatol Rep 2016;6:e206-e211.

\begin{abstract}
Objective The objective of this study was to evaluate the effect of a universal cervical length screening program on the incidence of antepartum interventions.

Study Design This retrospective cohort study included women delivering $\geq 20$ weeks of gestation with singleton pregnancies before and after implementing universal cervical length screening. Antepartum interventions included admission for threatened preterm birth, $\geq 2$ cervical length measurements, cervical cerclage, neonatology consultation, betamethasone, antibiotic administration for preterm premature rupture of membranes, and tocolysis.

Results There were 1,131 women-506 before the screening program (unexposed) and 625 afterward (exposed). The screening program resulted in significantly more women screened ( 3.0 vs. $69.9 \%, p<0.0001$ ). The exposed group was more likely to undergo $\geq 1$ intervention ( 20.0 vs. 9.5\%, $p<0.0001$ ); specifically, admission for threatened preterm birth (3.8 vs. $1.8 \%, p=0.04$ ) and $\geq 2$ cervical measurements

Keywords

- cervical length

- preterm birth

- antepartum interventions

- screening program

- birth outcomes (11.2 vs. $2.0 \%, p<0.001$ ). Other interventions were similar between groups (all $p \geq 0.06$ ). Median gestation length was significantly longer in the exposed (39.6 weeks [interquartile, IQR: 38.6-40.4] vs. 39.0 weeks [IQR: 38.0-40.0, $p<0.001]$ ); however, preterm delivery incidence was unaffected (9.4 vs. $10.9 \%, p=0.43$ ). Remaining neonatal outcomes were similar (all $p \geq 0.14$ ).

Conclusion Implementing universal cervical length screening significantly increased the proportion of women undergoing $\geq 1$ antepartum intervention. With the exception of a modestly prolonged gestation, other outcomes were unaffected.
\end{abstract}

Decreasing the incidence of preterm birth (delivery at $<37$ weeks of gestation) is an important public health goal, but one has been frustratingly difficult to accomplish. In 2000, the Department of Health and Human Services Healthy People 2010 campaign proposed an aim of decreasing the incidence of preterm birth from 11.6 to $7.6 \% .^{1}$ In the subsequent decade, tremendous effort had been dedicated to identifying and developing interventions to achieve such a reduction. Nevertheless, in 2011, the National Vital Statistics Report published a preterm delivery rate of $11.4 \%{ }^{2}$ Given these disappointing received

February 19, 2016

accepted after revision

April 11, 2016
DOI http://dx.doi.org/

10.1055/s-0036-1584240. ISSN 2157-6998.
Copyright $\odot 2016$ by Thieme Medical

Publishers, Inc., 333 Seventh Avenue, New York, NY 10001, USA. Tel: +1(212) 584-4662.
License terms

(요 (1) $\circledast$ 
results, ongoing efforts have been made to identify risk factors and preventive treatments for preterm delivery.

One of the strongest risk factors for preterm delivery is preterm delivery in a previous pregnancy. In addition, midtrimester cervical shortening has been shown to be a risk factor for preterm delivery, even in nulliparous women. ${ }^{3}$ Despite the use of vaginal progesterone to decrease the risk of spontaneous preterm birth in women with cervical shortening, there is no consensus on the utility of universal cervical length screening. ${ }^{4,5}$ A recent study found no difference in the incidence of preterm birth in low-risk women who underwent universal cervical length screening. ${ }^{6}$ Moreover, there is a paucity of data on the effect of universal cervical length screening programs on antepartum management and interventions.

Our objective was to evaluate the effect of a universal cervical length screening program on the incidence of antepartum interventions. We hypothesized that our transvaginal cervical length screening program would increase utilization of a composite of antepartum interventions, while having no effect on the incidence of preterm birth or gestational age at delivery.

\section{Methods}

In January 2012, our institution implemented a universal transvaginal ultrasound cervical length screening program in which women received cervical length screening at the time of the fetal anatomical ultrasound from 16 to 24 weeks of gestation. This was a retrospective cohort study comparing women who were eligible for cervical length screening after implementation of the screening program (exposed group) to women who were eligible before implementation of the screening program (unexposed group). We included all women who would have been eligible for cervical length screening and further limited to those with a singleton pregnancy who received prenatal care from providers referring to our ultrasound center, met gestational age criteria for cervical length screening (16-24 weeks of gestation), and delivered at $\geq 20$ weeks of gestation at our institution. The unexposed group included women whose full 16 to 24 weeks gestational age window occurred from January 1, 2011, to December 31, 2011. Women were included even if they did not receive their ultrasound at our unit, as there were no other universal transvaginal cervical length screening programs at our institution during the study period. Women in the unexposed group underwent cervical length screening based on obstetric history or symptoms at the discretion of the Maternal-Fetal Medicine specialists. To allow for transition to widespread utilization of the screening program (defined as two consecutive months in which $>80 \%$ of eligible women were screened), a 9-month washout period was incorporated into the study design. Thus, the exposed group included women whose full 16 to 24 weeks gestational age window occurred from October 1, 2012, to September 30, 2013. Both groups received their prenatal care from the same providers and offices during the entire study period. This study was approved by the Institutional Review Board at our institution.
As per guidelines of the screening program, cervical length measurements were obtained in standard transvaginal ultrasound fashion at the time of the mid-trimester fetal anatomical ultrasound and reviewed by a fellowship-trained Maternal-Fetal Medicine specialist. A short cervix was defined as $<20 \mathrm{~mm}$. Patients with a cervical length of $\geq 20 \mathrm{~mm}$ but $\leq 25 \mathrm{~mm}$ were scheduled for a repeat measurement in 1 to 2 weeks. Cervical cerclage was recommended to patients who had a cervical length of $<25 \mathrm{~mm}$ and a history of a spontaneous preterm birth or mid-trimester loss. In addition, cerclage was recommended for women without a history of preterm birth when their physical exam indicated cervical dilation before 24 weeks of gestation. Bed rest was not routinely prescribed; however, modified activity reduction was recommended at the discretion of the attending physician. There were no departmental guidelines for repeat cervical length measurements in patients with a short cervix with or without a cerclage; this was left to the discretion of the specialist.

The primary outcome was the occurrence of at least one of the following antepartum interventions: two or more cervical length measurements; cervical cerclage; neonatology consultation; admission for threatened preterm birth; or administration of betamethasone, antibiotics for preterm premature rupture of membranes, or tocolytics. A threatened preterm birth admission was included if it was for preterm premature rupture of membranes, preterm labor, threatened preterm labor, or cervical shortening/insufficiency. The secondary outcomes included each of the individual antepartum interventions, as well as the following birth and neonatal outcomes: gestational age at delivery, mode of delivery, birth weight, and Apgar scores. All data were extracted from the medical record and hospital databases.

This was a sample of convenience; thus, we included all eligible women as defined earlier. Data are presented as median (interquartile range) or proportion. Comparisons were made using a chi-square or Fisher exact test for categorical variables and the Wilcoxon rank-sum test for continuous variables. Log binomial regression was used to calculate risk ratios (RRs) and 95\% confidence intervals (CIs). Variables that were appreciably different between exposure groups and were known risk factors for the outcome were considered as potential confounders. They were assessed using univariate analysis and were considered for addition in the final model if they changed the RR by more than $10 \%$. To eliminate the potential for history-indicated cervical length screening, we repeated the analysis restricting to primigravid women. All tests were two sided, and $p$-values $<0.05$ were considered statistically significant. All analyses were performed with SAS 9.4 (SAS Institute Inc., Cary, NC).

\section{Results}

A total of 1,131 patients were included in this study; there were 625 (55.3\%) in the exposed group and 506 (44.7\%) in the unexposed group. Baseline demographic characteristics are presented in -Table 1. Maternal age and gravidity were similar; however, fewer women in the exposed (15.7\%) than unexposed group (22.7\%) identified as black. In the 
Table 1 Participant characteristics

\begin{tabular}{|c|c|c|c|c|}
\hline & \multicolumn{2}{|l|}{ Full cohort } & \multicolumn{2}{|c|}{ Primigravid cohort } \\
\hline & $\begin{array}{l}\text { Unexposed } \\
(n=506)\end{array}$ & $\begin{array}{l}\text { Exposed } \\
(n=625)\end{array}$ & $\begin{array}{l}\text { Unexposed } \\
(n=379)\end{array}$ & $\begin{array}{l}\text { Exposed } \\
(n=486)\end{array}$ \\
\hline Maternal age (y) & $28.9(24.7-32.1)$ & $29.2(25.5-32.7)$ & $28.9(24.5-31.9)$ & $29.3(25.3-32.4)$ \\
\hline \multicolumn{5}{|l|}{ Gravidity } \\
\hline 1 & $379(74.9)$ & $486(77.8)$ & $379(100)$ & $486(100)$ \\
\hline 2 & $86(17.0)$ & $99(15.8)$ & - & - \\
\hline 3 or more & $41(8.1)$ & $40(6.4)$ & - & - \\
\hline \multicolumn{5}{|l|}{ Race/ethnicity } \\
\hline Caucasian & $178(35.2)$ & $228(36.5)$ & $142(37.5)$ & 189 (38.9) \\
\hline Black & $115(22.7)$ & $98(15.7)$ & $78(20.6)$ & $76(15.6)$ \\
\hline Asian & $147(29.1)$ & $197(31.5)$ & $108(28.5)$ & $147(30.2)$ \\
\hline Hispanic & $46(9.1)$ & $49(7.8)$ & $36(9.5)$ & $37(7.6)$ \\
\hline Other/unknown & $20(4.0)$ & $53(8.5)$ & $15(4.0)$ & $37(7.6)$ \\
\hline $\begin{array}{l}\text { Any visit to ultrasound unit } \\
\text { from } 16 \text { to } 24 \mathrm{wk}\end{array}$ & $43(14.7)$ & $502(85.4)$ & $30(13.6)$ & $401(86.6)$ \\
\hline \multicolumn{5}{|l|}{ Number of cervical length measurements } \\
\hline 0 & $491(97.0)$ & $190(30.4)$ & $370(97.6)$ & $142(29.2)$ \\
\hline 1 or more & $15(3.0)$ & $435(69.6)$ & $9(2.4)$ & $344(70.8)$ \\
\hline Short cervix ( $<20 \mathrm{~mm}$ at initial measure) & $1(0.2)$ & $3(0.5)$ & $0(0.0)$ & $1(0.2)$ \\
\hline \multicolumn{5}{|l|}{ Mode of delivery } \\
\hline Cesarean & $165(32.6)$ & $170(27.2)$ & $113(29.8)$ & $134(27.6)$ \\
\hline Vaginal & $323(63.8)$ & $429(68.6)$ & $252(66.5)$ & $334(68.7)$ \\
\hline Operative vaginal & $18(3.6)$ & $26(4.2)$ & $14(3.7)$ & $18(3.7)$ \\
\hline
\end{tabular}

Note: Data are presented as median (interquartile range) or $n(\%)$.

exposed group, 435 women (69.6\%) had at least one transvaginal cervical length measurement; the remaining $30.4 \%$ either declined or were not offered screening. When restricting to women who presented to our center for an ultrasound from 16 to 24 weeks of gestation, 435 (86.7\%) of the exposed compared with 15 (34.9\%) of the unexposed group underwent at least one transvaginal cervical length measurement. There were three women $(0.5 \%)$ with a short cervix in the exposed group, while there was only one woman $(0.2 \%)$ in the unexposed group.

In our cohort, women in the exposed group were more likely to undergo at least one antepartum intervention (20.0\%) compared with the unexposed group (9.5\%). Race/ethnicity was the only variable that met our definition of a confounder. Although adjusting for race/ethnicity did not have a notable effect on the risk of women undergoing at least one antepartum intervention, it did change the RR for cerclage. Thus, all RRs presented are adjusted for race/ethnicity. Women in the exposed group were twice as likely to undergo at least one antepartum intervention (RR: 2.1, 95\% CI: 1.6-2.9). When the antepartum interventions were considered individually, we found that women in the exposed group were significantly more likely to have at least one repeat cervical length measurement (RR: 5.8, 95\% CI: 3.0-11.2) and a threatened preterm birth admission (RR: 2.2, 95\% CI: 1.04-4.8). The remaining antepartum interventions, including neonatology consultation and betamethasone, did not differ significantly between the two groups (all $p \geq 0.06$ ). Similarly, the incidence of preterm birth, low birth weight, and very low birth weight did not differ between the exposed and unexposed groups (all $p \geq 0.14$ ). However, the median gestational age at delivery was significantly greater in the exposed (39.6 weeks) than in the unexposed (39.0 weeks, $p<0.001$ ). Antepartum interventions and birth outcomes are presented in - Tables 2 and $\mathbf{3}$.

To further investigate the association between universal cervical length screening and threatened preterm birth admission, we calculated the incidence of admission separately for women who did and did not have a cervical length measurement. In contrast to what we observed in the full cohort, when restricting to women who had a cervical length measurement, the incidence of preterm birth admission was lower in the exposed group (2.3\%) compared with the unexposed group (13.3\%). Among women who did not have a cervical length measurement, the incidence was $7.4 \%$ in the exposed group and $1.4 \%$ in the unexposed group.

As with the full cohort, when restricting the analysis to primigravid women, we found women in the exposed group were significantly more likely to have two or more cervical 
Table 2 Antepartum interventions and neonatal outcomes

\begin{tabular}{|c|c|c|c|c|c|c|}
\hline & \multicolumn{3}{|l|}{ Full cohort } & \multicolumn{3}{|c|}{ Primigravid cohort } \\
\hline & $\begin{array}{l}\text { Unexposed } \\
(n=506)\end{array}$ & $\begin{array}{l}\text { Exposed } \\
(n=625)\end{array}$ & $p$-Value & $\begin{array}{l}\text { Unexposed } \\
(n=379)\end{array}$ & $\begin{array}{l}\text { Exposed } \\
(n=486)\end{array}$ & $p$-Value \\
\hline $\begin{array}{l}\text { At least one antepartum } \\
\text { intervention }{ }^{\mathrm{a}}\end{array}$ & $48(9.5)$ & $125(20.0)$ & $<0.001$ & $34(9.0)$ & $94(19.3)$ & $<0.001$ \\
\hline $\begin{array}{l}\text { Repeat }(\geq 2) \text { cervical } \\
\text { length measurement }\end{array}$ & $10(2.0)$ & $70(11.2)$ & $<0.001$ & $5(1.3)$ & $55(11.3)$ & $<0.001$ \\
\hline Cerclage placement & $4(0.8)$ & $3(0.5)$ & 0.71 & $2(0.5)$ & $0(0.0)$ & 0.19 \\
\hline Neonatology consult & $32(6.3)$ & $59(9.4)$ & 0.06 & $25(6.6)$ & $37(7.6)$ & 0.57 \\
\hline $\begin{array}{l}\text { Threatened preterm } \\
\text { birth admission }\end{array}$ & $9(1.8)$ & $24(3.8)$ & 0.04 & $5(1.3)$ & $13(2.7)$ & 0.17 \\
\hline $\begin{array}{l}\text { Total antepartum } \\
\text { hospital days }\end{array}$ & $4.5(3.6-16.4)$ & $5.7(3.4-9.6)$ & 0.67 & $4.5(2.8-14.5)$ & $4.3(3.9-7.2)$ & 0.70 \\
\hline \multicolumn{7}{|l|}{ Number of admissions } \\
\hline 0 & $497(98.2)$ & $601(96.2)$ & \multirow[t]{4}{*}{0.16} & $374(98.7)$ & $473(97.3)$ & \multirow[t]{4}{*}{0.10} \\
\hline 1 & $7(1.4)$ & $22(3.5)$ & & $3(0.8)$ & $12(2.5)$ & \\
\hline 2 & $1(0.2)$ & $1(0.2)$ & & $1(0.3)$ & $0(0.0)$ & \\
\hline 3 & $1(0.2)$ & $1(0.2)$ & & $1(0.3)$ & $1(0.2)$ & \\
\hline \multicolumn{7}{|l|}{ Medications } \\
\hline Betamethasone & $18(3.6)$ & $29(4.6)$ & 0.36 & $14(3.7)$ & $16(3.3)$ & 0.75 \\
\hline Antibiotics & $4(0.8)$ & $12(1.9)$ & 0.11 & $3(0.8)$ & $8(1.6)$ & 0.36 \\
\hline Tocolytics & $12(2.4)$ & $19(3.0)$ & 0.49 & $8(2.1)$ & $15(3.1)$ & 0.38 \\
\hline $\begin{array}{l}\text { Gestational age at } \\
\text { delivery }\end{array}$ & $39.0(38.0-40.0)$ & $39.6(38.0-40.0)$ & $<0.001$ & $39.0(38.0-40.0)$ & $39.6(38.6-40.4)$ & $<0.001$ \\
\hline \multicolumn{7}{|l|}{ Preterm birth } \\
\hline$<37$ wk & $55(10.9)$ & $59(9.4)$ & 0.43 & $41(10.8)$ & $42(8.6)$ & 0.28 \\
\hline$<34$ wk & $14(2.8)$ & $23(3.7)$ & 0.39 & $11(2.9)$ & $15(3.1)$ & 0.88 \\
\hline$<32$ wk & $12(2.4)$ & $12(1.9)$ & 0.60 & $9(2.4)$ & $8(1.6)$ & 0.44 \\
\hline $\begin{array}{l}\text { Low birth weight } \\
(<2,500 \mathrm{~g})\end{array}$ & $34(6.7)$ & $57(9.1)$ & 0.14 & $27(7.1)$ & $41(8.4)$ & 0.48 \\
\hline $\begin{array}{l}\text { Very low birth weight } \\
(<1,500 \mathrm{~g})\end{array}$ & $6(1.2)$ & $11(1.8)$ & 0.43 & $5(1.3)$ & $7(1.4)$ & 0.88 \\
\hline
\end{tabular}

Note: Data are presented as median (interquartile range) or $n(\%)$.

${ }^{a}$ Defined as having one or more of the following: two or more cervical length measurements; cervical cerclage; neonatology consultation; admission for threatened preterm birth; or administration of betamethasone, antibiotics for preterm premature rupture of membranes, or tocolytics.

${ }^{\mathrm{b} C a l c u l a t e d}$ for only those with an antepartum admission.

length measurements (11.3\%) than in the unexposed group $(1.3 \%, p<0.001)$. Although the incidence of threatened preterm birth admission remained higher in the exposed group $(2.7 \%)$ than in the unexposed group (1.3\%), this difference was not statistically significant $(p=0.17)$.

\section{Discussion}

As hypothesized, our universal transvaginal cervical length screening program was associated with a twofold increase in antepartum interventions. Specifically, the exposed group was significantly more likely to undergo two or more transvaginal cervical length measurements and be admitted for threatened preterm birth. However, screening did not appear to affect the incidence of any of the other interventions, including length of admission, neonatology consultation, and betamethasone administration. There was a significant difference (0.6 weeks) in the gestational age at delivery between the exposed and unexposed groups; however, it is unlikely that this difference is clinically meaningful. There were no differences in other birth outcomes between the two groups. Although there were relatively few women in the unexposed group who had an ultrasound from 16 to 24 weeks at our ultrasound unit, we believe this group still serves as an appropriate comparison group as there was no routine transvaginal cervical length screening at our institution during that time period. Contrary to what we expected, the association between universal cervical length screening and 
Table 3 Risk of antepartum interventions

\begin{tabular}{|c|c|c|}
\hline & \multicolumn{2}{|l|}{ Full cohort } \\
\hline & Crude risk ratio $(95 \% \mathrm{Cl})$ & Adjusted $^{\mathrm{a}}$ risk ratio $(95 \% \mathrm{Cl})$ \\
\hline At least one antepartum intervention ${ }^{\mathrm{b}}$ & $2.1(1.5-2.9)$ & $2.1(1.6-2.9)$ \\
\hline Repeat ( $\geq 2$ ) cervical length measurement & $5.7(3.0-10.9)$ & $5.8(3.0-11.2)$ \\
\hline Cerclage placement & $0.61(0.14-2.7)$ & $0.70(0.16-3.2)$ \\
\hline Neonatology consult & $1.5(0.99-2.3)$ & $1.5(0.999-2.3)$ \\
\hline Threatened preterm birth admission & $2.2(1.01-4.6)$ & $2.2(1.04-4.8)$ \\
\hline \multicolumn{3}{|l|}{ Medications } \\
\hline Betamethasone & $1.3(0.73-2.3)$ & $1.4(0.78-2.5)$ \\
\hline Antibiotics & $2.4(0.79-7.5)$ & $2.4(0.78-7.5)$ \\
\hline Tocolytics & $1.3(0.63-2.6)$ & $1.4(0.67-2.8)$ \\
\hline
\end{tabular}

Abbreviation: $\mathrm{Cl}$, confidence interval.

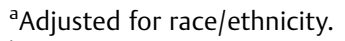

bDefined as having one or more of the following: two or more cervical length measurements; cervical cerclage; neonatology consultation; admission for threatened preterm birth; or administration of betamethasone, antibiotics for preterm premature rupture of membranes, or tocolytics.

threatened preterm birth admission was driven by women who did not have a cervical length measurement. In the exposed group, the incidence of admission was only $2.3 \%$ among women who had a cervical length measurement and $7.4 \%$ among those who did not. This could be explained by chance or the perception that women who did not receive screening had an inherently higher risk of threatened preterm birth, which resulted in a lower threshold for admission.

Not surprisingly, among the full cohort, there were no differences in the administration of tocolytics or antibiotics for preterm premature rupture of membranes, as the exposure of cervical length screening should not confer an increased risk of either. We had anticipated that knowledge of the cervical length would result in obstetric providers having a lower threshold for low-risk interventions, such as betamethasone and neonatology consultation. However, we did not find a significant difference in these interventions. This could be explained by our lack of power to detect differences of the observed magnitude. Our post hoc power calculations demonstrated we had only 49 and $14 \%$ power to detect the observed differences in neonatology consultation and betamethasone administration, respectively.

In terms of birth outcomes, we did not demonstrate any difference in the incidence of preterm birth at $<37,<35$, or $<32$ weeks of gestation. As second-trimester cervical shortening is an independent risk factor for spontaneous preterm birth, this finding is not surprising given the low incidence of cervical shortening was similar between the two groups. ${ }^{3,4,7}$ In a recent prospective trial evaluating a universal cervical length screening program, Orzechowski et al, too, found no difference in the incidence of spontaneous preterm birth between the screened and nonscreened groups, raising the question of benefit with universal screening. ${ }^{6}$ Our data lend support to these findings. In addition, in a recent cohort of low-risk asymptomatic women undergoing routine cervical length screening, it was determined that 675 women needed to be screened to prevent one preterm birth. ${ }^{8}$ Given this, we are not surprised that there were no differences in the rate of preterm birth between our two groups. Furthermore, a recent Cochrane Review concluded that there is insufficient evidence to support universal cervical length screening to prevent preterm birth. ${ }^{9}$ However, providers may struggle with balancing data such as ours and that of Orzechowski et al with potential missed opportunities to start vaginal progesterone in women who would potentially benefit. At this point, large prospective randomized controlled trials are needed to determine the utility and cost effectiveness of universal cervical length screening.

To our knowledge, this is the first study investigating the effect of a universal cervical length screening program on antepartum interventions. Our study has several strengths. The study design included a 9-month washout period to allow for $>80 \%$ acceptance of cervical length screening among women presenting for ultrasound. The widespread acceptance of our screening program is greater than that in previously published reports. ${ }^{10,11}$ In addition, each antepartum intervention, as well as the indication for admission, was confirmed by medical record review. Finally, while other studies have presented results on nulliparous women only, our analysis restricted to primigravid women further limits any effect of obstetrical history on the management of the index pregnancy.

One limitation of the study is that some women in the unexposed group did in fact have transvaginal cervical length screening. At that time, the clinical practice was to screen women based on obstetrical history, history of surgical cervical procedures, or symptomatology, at the discretion of the Maternal-Fetal Medicine specialist. In addition, given the retrospective nature of the study, patients and providers were not blinded to the length of the cervix, which may have influenced decision making around certain antepartum interventions. However, we do not believe this played a role in our findings as women in the exposed group who did not have a cervical length measurement had a higher incidence of threatened preterm birth admission than women who did have a cervical length measurement. Although our standard practice is to recommend vaginal progesterone to women with a short cervix, we could not accurately 
assess the use of vaginal progesterone within this cohort. Finally, these findings could be limited by our sample size.

In conclusion, our data support our hypothesis that the presence of a universal cervical length screening program increases the utilization of antepartum interventions. We demonstrated a significant increase in the proportion of women undergoing repeat transvaginal cervical length screening, as well as threatened preterm birth admission; however, it is unclear whether the increase in admissions is a result of the screening program itself. Despite these findings, we failed to show a clinically meaningful improvement in birth outcomes, leading us to question the utility of universal screening. These findings have resulted in a departmental policy change eliminating universal transvaginal cervical length screening. Future studies are needed to identify subgroups of women who would benefit from universal cervical length screening.

\section{Conflict of Interest}

The authors report no conflict of interest.

Note

This work was presented at the 2015 annual meeting of the New England Perinatal Society, Newport, RI, March 13-15, 2015.

\section{Acknowledgments}

This work was conducted with support from Harvard Catalyst, The Harvard Clinical and Translational Science Center (National Center for Research Resources and the National Center for Advancing Translational Sciences, National Institutes of Health Award UL1 TR001102) and financial contributions from Harvard University and its affiliated academic healthcare centers.

\section{References}

1 Center for Disease Control and Prevention. National Center for Health Statistics. Healthy People 2010. 2012. Available at: http:// www.cdc.gov/nchs/data/hpdata2010/hp2010_final_review.pdf. Accessed April 15, 2015

2 Martin JA, Hamilton BE, Ventura SJ, Osterman MJ, Mathews TJ. Center for Disease Control and Prevention. Births: final data for 2011. Natl Vital Stat Rep 2013;62(1):1-69, 72

3 Iams JD, Goldenberg RL, Meis PJ, et al; National Institute of Child Health and Human Development Maternal Fetal Medicine Unit Network. The length of the cervix and the risk of spontaneous premature delivery. N Engl J Med 1996;334(9):567-572

4 Berghella V; Society for Maternal-Fetal Medicine Publications Committee, with assistance of Vincenzo Berghella. Progesterone and preterm birth prevention: translating clinical trials data into clinical practice. Am J Obstet Gynecol 2012;206(5): 376-386

5 Parry S, Simhan H, Elovitz M, Iams J. Universal maternal cervical length screening during the second trimester: pros and cons of a strategy to identify women at risk of spontaneous preterm delivery. Am J Obstet Gynecol 2012;207(2):101-106

6 Orzechowski KM, Boelig R, Nicholas SS, Baxter J, Berghella V. Is universal cervical length screening indicated in women with prior term birth? Am J Obstet Gynecol 2015;212(2):234.e1-234.e5

7 Iams JD, Goldenberg RL, Mercer BM, et al; National Institute of Child Health and Human Development Maternal-Fetal Medicine Units Network. The Preterm Prediction Study: recurrence risk of spontaneous preterm birth. Am J Obstet Gynecol 1998;178(5): 1035-1040

8 Miller ES, Sakowicz A, Grobman WA. Association between secondtrimester cervical length and primary cesarean delivery. Obstet Gynecol 2013;122(4):863-867

9 Berghella V, Baxter JK, Hendrix NW. Cervical assessment by ultrasound for preventing preterm delivery. Cochrane Database Syst Rev 2013;1(1):CD007235

10 Orzechowski KM, Boelig RC, Baxter JK, Berghella V. A universal transvaginal cervical length screening program for preterm birth prevention. Obstet Gynecol 2014;124(3):520-525

11 Orzechowski KM, Nicholas SS, Baxter JK, Weiner S, Berghella V. Implementation of a universal cervical length screening program for the prevention of preterm birth. Am J Perinatol 2014;31(12): 1057-1062 